\title{
El juego como estrategia pedagógica para la enseñanza de las matemáticas: retos maestros de primera infancia*
}

\section{The game as a pedagogical strategy for teaching of mathematics: Early childhood master challenges}

\author{
Adriana María Gallego Henao ${ }^{1}$, Enid Daniela Vargas Mesa² ${ }^{(D)}$, Oscar Alberto Peláez Henao ${ }^{(\mathbb{D})}$, \\ Leisy Magdali Arroyave Taborda ${ }^{(\mathbb{D})}$, Leidy Johana Rodríguez Marín ${ }^{5}$
}

Para citar este artículo: Gallego, A. M., Vargas, E. D., Pe-

Recibido: 21-10-2019 - Aceptado: 06-07-2020

láez, O. A., Arroyave, L. M., Rodríguez, L. J. (2020). El jue-

go como estrategia pedagógica para la enseñanza de las matemáticas: retos maestros de primera infancia. Infancias

Imágenes, 19(2), 133-142

\section{Resumen}

El objetivo de este artículo es describir la incidencia del juego como estrategia pedagógica y su relación con el aprendizaje de las nociones lógico-matemáticas. El enfoque metodológico fue cualitativo y se hizo énfasis en la estrategia del estudio de caso intrínseco. Se encontró que el juego es un medio de expresión y comunión en la infancia y que desde el lugar del aula este se puede emplear como una estrategia pedagógica que favorece los aprendizajes de las matemáticas. Se concluye que el juego, al ser comprendido como estrategia pedagógica, debe estar encaminado por los docentes como manera de aprender y divertirse al mismo tiempo; en especial cuando se quiere enseñar un concepto matemático. Palabras clave: aprendizaje, juego, pensamiento, matemáticas.

* El presente artículo de reflexión es derivado del proyecto de investigación "Los maestros como agentes potenciadores de los procesos lógico-matemáticos". Iniciada el 1 febrero de 2018 y finalizada en diciembre de 2018. Este producto de investigación se articula al grupo Educación, Infancia y Lenguas Extranjeras.

1 Magister en Educación y Desarrollo Humano. Coordinadora de la Maestría en Educación. Docente investigadora de la Universidad Católica Luis Amigó. Integrante del grupo de investigación Educación, Infancia y Lenguas Extranjeras. Universidad Católica Luis Amigó, MedellínColombia. Correo electrónico: adriana.gallegohe@amigo.edu.co. Orcid: https://orcid.org/0000-0003-2952-1603

2 Joven investigadora de la Facultad de Educación y Humanidades. Especialista en Docencia Universitaria. Docente de la Universidad Católica Luis Amigó. Integrante del grupo de investigación Educación, Infancia y Lenguas Extranjeras. Aspirante al título de magister en Educación. Universidad Católica Luis Amigó, Medellín-Colombia. Correo electrónico: enid.vargasme@amigo.edu.co. Orcid: https://orcid. org/0000-0003-2228-2072

3 Magíster en Educación. Líder de la línea Construcciones Investigativas en Lenguas Extranjeras. Docente investigador y coordinador del campo investigativo Licenciatura en Inglés, Universidad Católica Luis Amigó, Medellín-Colombia. Correo electrónico: oscar.pelaezhe@ amigo.edu.co. Orcid: https://orcid.org/0000-0002-3202-036X

4 Magíster en Adicciones. Coordinadora de las Especializaciones en Educación. Docente investigadora de la Universidad Católica Luis Amigó. Integrante del grupo de investigación Educación, Infancia y Lenguas Extranjeras. Universidad Católica Luis Amigó, MedellínColombia. Correo electrónico: leisy.arroyaveta@amigo.edu.co. Orcid: https://orcid.org/0000-0003-3982-2805

5 Licenciada en Educación Preescolar, Universidad Católica Luis Amigó, Medellín-Colombia. Correo electrónico: leiidy.rodriguez29@gmail. com. Orcid: https://orcid.org/0000-0002-0064-643X 
the classroom it can be used as a pedagogical strategy that favors learning mathematics. It is concluded that the game, when understood as a pedagogical strategy, must be directed by the teachers as a way to learn and have fun at the same time, especially when you want to teach a mathematical concept.

Keywords: learning, game, mathematical thought.

\section{Introducción}

El juego es visto socialmente como una estrategia de diversión o de entretenimiento en la cual los niños en ocasiones pierden el tiempo. Sin embargo, desde el ámbito educativo y pedagógico se ha demostrado que los niños ${ }^{1}$ adquieren aprendizajes significativos, desarrollo de competencias y habilidades sociales cuando juegan. Es por ello por lo que en las aulas de clase $y$, especialmente en la primera infancia, el juego es la estrategia privilegiada para la enseñanza de las nociones o conceptos. García y Llull expresan que "a lo largo de las etapas de Educación Infantil y Primaria, el juego aparece como un instrumento natural para la maduración en todas las dimensiones de la persona; es decir, los niños y las niñas aprenden jugando" (2009, p. 316), aunque no jueguen con la intención de aprender.

Vemos entonces que los niños juegan constantemente en su día a día, en los diferentes lugares en que se encuentran y van incorporando diversos juegos según la etapa de desarrollo en la que se encuentre, como, por ejemplo, el juego simbólico, de reglas, construcción, entre otros. Cabe resaltar que el juego produce diferentes emociones y sentires en la vida de los niños, debido a que trae consigo disfrute y placer; además, de permitirle interiorizar aprendizajes no solo teóricos sino relaciónales. Al respecto García y Llull expresan que "es fundamental para todos los profesionales de la educación conocer en profundidad la importancia del juego, sus características y las posibilidades psicopedagógicas del modelo lúdico" (2009, p. 316). Por medio del juego se pueden potenciar diferentes experiencias o conocimientos en los niños teniendo en cuenta que desde bebés gozan de este de manera genuina.

1 En el texto se usará la palabra niño por facilidades en el lenguaje, pero se aclara que en esta categoría están incluidas las niñas.
El juego es una estrategia pedagógica que promueve múltiples aprendizajes y le permite al niño conocer, investigar, experimentar, descubrir su contexto de una manera amigable y lúdica. En este sentido, Chamoso et al. refieren que "los juegos se utilizan a cualquier edad pues las ventajas de aprender en un ambiente agradable son independientes de ésta" (2004, p. 50). Lo anterior sugiere que a través del juego los niños pueden comunicar diferentes necesidades y situaciones que les ayudan a desarrollar habilidades cognitivas, físicas y sociales como, por ejemplo, a diferenciar objetos, números, colores, a conocer su espacialidad, a relacionarse con sus pares, entre otras.

A través de la práctica docente se ha podido observar que algunos agentes educativos ${ }^{2}$, entre ellos algunos maestros, incorporan el juego en sus aulas de clase solo como un estímulo. Es decir, utilizan el juego para condicionar ciertos comportamientos como, por ejemplo, decirles "si permaneces sentado y juicioso en esta actividad puedes ir a jugar", "como hiciste tus deberes puedes ir a jugar" o "como no hiciste tus deberes no puedes jugar". Estas expresiones denotan cierto desconocimiento sobre las bondades del juego en la cotidianidad de la vida de los niños y de la escuela, a lo que comentan Chamoso et al. que "los juegos son un recurso didáctico más y, como cualquier otro instrumento, debe incorporarse al aula de un modo meditado y planificado, con una programación previa que tenga en cuenta todos los factores del proceso de enseñanza-aprendizaje" (2004, p. 51).

Ahora bien, la enseñanza de las matemáticas en la primera infancia está enmarcada por una serie de mitos que se han transmitido de generación en

2 Este término según el Ministerio de Educación Nacional es usado para referirse a padres, madres, maestros o cualquier persona significativa que tiene contacto con los niños. 
generación. Así como lo afirman Callejo y Vila, las investigaciones sobre las creencias de los alumnos nos muestran que la visión de la matemática que predomina es la de una ciencia rígida, aburrida, mecánica, difícil, un tormento para algunos, que poco o nada tiene que ver con la creatividad, la belleza o el juego (2003, p. 173).

Es importante resaltar que las matemáticas no se limitan solo a fichas, estas son solamente una de las muchas estrategias que existen para potenciar el desarrollo de las nociones lógico-matemáticas. A partir de algunas conversaciones con maestros se puede señalar que estas se refieren a las fichas como únicas actividades que se revisten de importancia en la enseñanza de las matemáticas, dejando de lado los diferentes juegos que coadyuvan a los niños en el desarrollo de los aprendizajes de esta área.

Lo dicho hasta aquí supone que a través del juego se puede iniciar la enseñanza de las nociones lógico-matemáticas. Esto es debido a que se trata de una estrategia pedagógica que favorece (a través del disfrute) el desarrollo de nociones matemáticas como: temporalidad, espacialidad, seriación entre otros conocimientos. Posada et al. afirman que el juego:

Es la principal actividad del niño; estimula su desarrollo motor, social, emocional y cognoscitivo, y favorece el desarrollo de la creatividad, la solidaridad y la felicidad. Jugar es una necesidad para el niño; así se pone en contacto con la realidad, libera tensiones, adquiere habilidades y destrezas, aprende a afrontar situaciones nuevas, imita a los demás, conoce los diferentes roles y da rienda suelta a su imaginación y creatividad. (2005, p. 217)

Y definen el juego infantil como "actividad placentera, libre y espontánea sin un fin determinado, pero de gran utilidad para el desarrollo del niño" (2005, p. 316). De allí parte la necesidad y la importancia de que docentes incorporen al juego a sus clases como elemento pedagógico imprescindible.

Ahora bien, es necesario abordar el tema de las nociones lógico-matemáticas en la primera infancia. Para esto es menester recordar que las matemáticas no solo se evidencian y se aprenden en las aulas, sino que se encuentran inmersas en el diario vivir. "Las relaciones que tienen los niños con el conocimiento lógico-matemático son en un primer momento sensomotoras, luego intuitivas y finalmente lógicas, según su nivel de desarrollo y se expresarán mediante la acción, el lenguaje oral y finalmente el matemático" (Blanco, s. f., p. 5); desde que nacen, los niños pasan por diferentes etapas de desarrollo, las cuales van desde lo micro a lo macro, facilitando su maduración intelectual.

Tal y como lo expresa Blanco se debe tener en cuenta que la "iniciación matemática ha de ser una construcción mental vivida y experimentada. Debe estar motivada mediante los materiales manipulativos, debe lograr una progresiva asunción de los conceptos matemáticos, de modo que se consiga un dominio de ellos en las actividades cotidianas" (s. f., p. 6). Es por esto por lo que es posible motivar a los niños para que aprendan a través del juego y puedan desarrollar diferentes pensamientos matemáticos en actividades cotidianas como manipular, observar, realizar y experimentar con todo lo que se encuentra en su entorno.

Lo mencionado hasta aquí dio origen a la pregunta de investigación: ¿cuál es la relación que existe entre el juego como estrategia pedagógica y el desarrollo de las nociones lógico-matemáticas en la primera infancia?

Este artículo permite reconocer el juego como estrategia pedagógica en el aula de clase y desde este fortalecer las competencias de los niños para aprender nociones matemáticas, las cuales determinan en los posteriores años el desarrollo de conceptos matemáticos y algebraicos.

El contenido del artículo se centra en los siguientes apartados: el juego como medio de expresión y comunicación en la infancia; el juego: una estrategia que favorece los aprendizajes en la infancia; el juego y su relación con la matemática; juegos que favorecen el aprendizaje de las nociones matemáticas; juegos didácticos y su aporte el desarrollo de los aprendizajes.

\section{Metodología}

El paradigma en el que se desarrolló el proyecto de investigación responde al cualitativo desde un enfoque hermenéutico; en tanto que este tipo de estudios, según Grondin, permite al investigador 
"entender el discurso tan bien como el autor, y después mejor que él" (1999, p. 107). En este sentido, esta apuesta investigativa desde la hermenéutica pretende develar las experiencias y vivencias que enmarcan las practicas pedagógicas de los maestros y agentes educativos que trabajan por la primera infancia.

El carácter del estudio fue descriptivo, en el que se hizo énfasis en el estudio de caso intrínseco. Este, según Galeano (2004), permite una mejor comprensión del fenómeno en el que priman la historia, vivencias, experiencias y las características de los contextos y sus participantes; así es como se pasa a un segundo plano la construcción de teoría.

Se resalta que el estudio se llevó a cabo en tres fases: 1) exploración, en la que se sostuvo acercamientos preliminares con los participantes; 2) focalización, el interés se centró en la aplicación de los instrumentos y generación de la información; 3) profundización, en la cual se realizó el análisis e interpretación de la información recolectada. De

136 igual manera, y en coherencia con las consideraciones éticas del estudio, en esta tercera fase se programó la devolución y validación de los resultados con los participantes.

\section{Universo y participantes}

El escenario de esta investigación fue una Institución Educativa de la comuna 11 en el barrio Estadio. Esta institución de educación formal actualmente ofrece varios niveles educativos como preescolar, básica primaria, básica secundaria y media académica. Es una institución orientada por la educación católica. Los participantes fueron 14 niñas y dos docentes pertenecientes al grado prejardín.

\section{Técnicas de generación de información y de sistematización}

Para el desarrollo de este aspecto se combinaron técnicas de generación de información tales como: 1) observaciones no participantes, las cuales fueron materializadas en cinco observaciones registradas en sus respectivos diarios de campo; 2) entrevistas semiestructurada, se aplicaron dos a las dos docentes; 3) técnicas interactivas, como mural de situaciones, el árbol de problemas y siluetas, entre otras. En total se aplicaron nueve técnicas con las estudiantes. Con relación a las técnicas de registro de información se realizaron diarios de campo, guías de observación y trascripciones.

Luego de registrar la información se procedió a organizarla en matrices categoriales con las cuales se buscaron las tendencias del estudio para posteriormente agrupar los datos en categorías y proceder con la escritura de la información

\section{Resultados y discusión}

\section{El juego como medio de expresión y comunicación en la infancia}

El juego, es una estrategia mediante la cual los niños logran adquirir una serie de conocimientos de manera intencional y no intencional. Cuando los niños parten de sus intereses logran adquirir conocimientos de manera significativa; este se convierte en un aprendizaje para la vida, pues "Ios niños a través de sus propias experiencias sensoriales: mirar, tocar, oír, probar, oler, formarán las bases de su entendimiento para más tarde alcanzar el desarrollo de ideas y tareas más complejas" (Bedolla, s. f., p. 2). Es por esta razón que se hace necesario resignificar el concepto que le están dando los docentes de primera infancia al juego como eje potencializador del aprendizaje. Debido a que, tal y como lo describe Bedolla:

Conforme el niño va evolucionando comienza a destacar una de las actividades infantiles más importantes y naturales para el niño en esta etapa: jugar. Jugar es para los niños uno de los modos principales de experimentar el mundo, por lo cual el juego ha sido Ilamado trabajo de la infancia. (s. f., p. 2)

De acuerdo con lo expresado por el autor, el juego es una actividad fundamental en la infancia porque es la manera en la que los niños se expresan y exploran su propio entorno, además de permitirles disfrutar y conocer el mundo en el cual están inmersos. Cárdenas y Gómez establecen que "el juego es reflejo de la cultura, de las dinámicas sociales de una comunidad, y en él las niñas y los niños representan las construcciones y desarrollos de su vida y contexto" (2014, p. 12). Es así como el juego ha de ser visto como una estrategia que le permita a las personas cercanas al niño descubrir 
sus necesidades e intereses a la hora de jugar $y$, además, reconocer su mundo interior.

Ahora bien, "El juego es una actividad del niño que produce una expansión del sí mismo, permite experimentar distintas posibilidades, implicando el dominio de las acciones y la presencia de algún elemento sorpresa (se produce una conquista)" (Schejtman et al., 2009, p. 244). A través del juego logran canalizar sus pensamientos, emociones, además, transmiten sus intereses y necesidades.

\section{El juego: una estrategia que favorece los}

\section{aprendizajes en la infancia}

Por otro lado, el cuerpo es un elemento fundamental al momento de jugar, debido a que las primeras interacciones que se dan en la etapa inicial se desarrollan por medio de este. Para Camels, los juegos que se realizan con los bebés se desarrollan primero desde las sensaciones y la estimulación que se hace con ellos, esto les permite a los niños reconocer su corporeidad e "implica esencialmente tomar y poner el cuerpo como objeto y motor del jugar" (2010, p. 1). Todo ello implica el punto de partida que le permite al niño reconocerse en su entorno y realizar múltiples actividades, entre ellas jugar.

De este modo, el docente brinda la confianza necesaria que requiere el niño para adaptarse al entorno en el cual se encuentra y que le permite adquirir seguridad y confianza en dicho contexto. Tal y como lo expresan Quintero et al. "el juego realmente es una herramienta que no puede faltar en el ejercicio profesional de la docente de primera infancia, quien puede generar, para los niños, ambientes de aprendizaje agradables, motivantes y cargados de lúdica" (2016, p. 165).

Ahora bien, los niños al experimentar y descubrir las habilidades que tienen con su cuerpo, como moverse, balancearse, tocar, entre otros, se pasan a una etapa en la cual lo emplean "de manera más activa e independiente, en saltos, deslizamientos, lanzamientos, carreras, persecuciones y acciones más estructuradas que conforman juegos y rondas" (Cárdenas y Gómez, 2014, p. 15). Esto quiere decir que el niño emplea su propio cuerpo para desplazarse y explorar el mundo del cual hacen parte y, en simultáneo, aprende nuevos conceptos que pueden ser relevantes para su vida, entre ellas la socialización con sus pares y adultos significativos.

Asimismo, Vallejo sostiene que "Ilamamos juego a la actitud con la que se hace algo: libertad, espontaneidad, complicidad, gratuidad, búsqueda de placer. Sin placer y autosatisfacción por lograr lo que se ha propuesto el propio jugador/a, no hay juego" (p. 197). En este orden de ideas "el juego es una experiencia siempre creadora, y es una experiencia en el continuo espacio-tiempo. Una forma básica de vida" (Winnicott, 1982, p. 75). Es decir, el juego es una actividad creadora y recreadora en el cual los niños descubren, imaginan y desarrollan habilidades corporales y las particularidades de su entorno y los objetos con los cuales interfieren al momento de jugar.

El Ministerio de Educación Nacional afirma que "el juego es un lenguaje natural porque es precisamente en esos momentos lúdicos en los que la niña y el niño sienten mayor necesidad de expresar al otro sus intenciones, sus deseos, sus emociones y sus sentimientos" (MEN, 2014, p. 15). Por lo tanto, es necesario que en todos los ámbitos en los que se desenvuelve el niño haya múltiples espacios para el juego.

\section{El juego y su relación con la matemática}

Chacón (2009) expresa que "la relación entre juego y aprendizaje es natural; los verbos 'jugar' y 'aprender' confluyen. Ambos vocablos consisten en superar obstáculos, encontrar el camino, entrenarse, deducir, inventar, adivinar y llegar a ganar... para pasarlo bien, para avanzar y mejorar" (p. 2). Así pues, el docente debe aprovechar las posibilidades que tiene el juego como una herramienta que brinda la oportunidad de divertirse y adquirir habilidades de manera simultánea.

Es así como el maestro dinamizador del aprendizaje, por medio del juego, está Ilamado a generar interacciones texto-contexto-estudiante. Ello posibilitará que las clases estén permeadas por el factor motivación; los niños al estar motivados estarán más conectados con los contenidos y sus avances académicos se verán reflejados en los desempeños académicos. Quintero et al. (2016) afirman que: 
Se requiere entonces de un maestro que se presente coherente en su ser y en su hacer, para favorecer una trasferencia positiva en la labor pedagógica con los niños con el fin de generar el deseo de conocer, de explorar y de aprender. (p. 168)

Además de esto, es importante que el docente plantee los objetivos que permitan lograr los aprendizajes esperados por sus aprendices. De acuerdo con Chacón (2009), estas metas deben establecerse de la siguiente manera:

Plantear un problema que deberá resolverse en un nivel de comprensión que implique ciertos grados de dificultad. Afianzar de manera atractiva los conceptos, procedimientos y actitudes contempladas en el programa. Ofrecer un medio para trabajar en equipo de una manera agradable y satisfactoria. Reforzar habilidades que el niño necesitará más adelante. (p. 3)

Asimismo, es importante que se genere un ambiente de aprendizaje que estimule la creatividad y el desarrollo de las emociones en tanto estos aspectos son esenciales para que se activen los dispositivos básicos de aprendizaje, y es el juego el motor o canal. Para ello, cabe resaltar que:

El juego se potencia dependiendo de las condiciones de contexto, se orienta según la cultura y las costumbres y se vive de acuerdo con los saberes específicos de cada territorio, del grupo poblacional, de las niñas, los niños, las maestras, los maestros y agentes educativos. (p. 21)

En este aspecto Salvador (2010), plantea que: "la mejor manera de despertar el interés y el deseo de descubrir a los alumnos es presentando un juego, una paradoja, un truco de magia o una experiencia" (p. 18). Lo anterior resalta la importancia en la selección de las estrategias para el desarrollo de los contenidos que se espera aprendan los niños. Es así que el éxito del tema siempre dependerá de la forma como se introduzca al mismo; es necesario vincular desde el inicio a los niños con el tema y un buen juego puede servir para introducirlo o afianzar el desarrollo de este. Los niños juegan a lo que ven y "juegan lo que viven resignificándolo, por esta razón el juego es considerado como una forma de elaboración del mundo y de formación cultural, puesto que los inicia en la vida de la sociedad en la cual están inmersos" (MEN, 2014, p. 14). Como maestro es necesario que se despierte el entusiasmo e interés del estudiante incluso desde la misma salud, esto elevará de inmediato la motivación y el deseo por aprender.

Es así como el juego permite a los niños un mayor acercamiento a conceptos más complejos a los que se enfrentarán en su vida escolar. Muñoz y Almonacid establecen que "el juego es lenguaje primitivo y profundo, es la historia de los niños y niñas que inmersos en el mundo de los adultos escapan en una especie de catarsis liberadora y mágica" (2015, p. 167). De ello se destaca la importancia de atraerlos hacia dichas nociones de manera agradable y que puedan ir comprendiendo poco a poco, a medida que van adquiriendo madurez cognitiva de acuerdo con sus intereses.

En estas circunstancias es importante que el maestro propicie experiencias a los estudiantes que le permitan lograr un aprendizaje significativo de las matemáticas a partir de las vivencias cotidianas. En este sentido, Bustamante (2004) refuerza esta afirmación de la siguiente manera:

Cobra importancia la consideración del poder que tienen las estrategias de enseñanza que el docente propone, que involucran las actividades de carácter cognitivo-procedimental que realiza el niño/ niña en los primeros años de su etapa escolar, y que pretenden el desarrollo del pensamiento en general y del lógico-matemático en particular. Nos referiremos aquí, a las experiencias que buscan desarrollar la capacidad para organizarse en el espacio mediante el fomento de relaciones de características lógico-matemáticas, que el niño/niña establece con su medio a través de las experiencias que cotidianamente vive. (2004, p. 163)

Así las cosas, Bustamante (2004) señala que la enseñanza de la lógica matemática en los niños de edad preescolar debe basarse en aquello que los niños cotidianamente conocen y tienen a su alrededor. De este modo, se hace una enseñanza 
contextualizada que permite que el estudiante retenga fácilmente aquello que se le desea enseñar. Asimismo, los niños en esta etapa escolar presentan mucha actividad física y a través de esto están en constante interacción con el entorno que le rodea. Es así que el niño construye y desarrolla el pensamiento; y es responsabilidad del docente aprovechar e indagar actividades que permitan tal acercamiento que contribuyan al aprendizaje de los conceptos matemáticos simples y posteriormente complejizarlos en la medida en la que el niño adquiere madurez cognitiva.

\section{Juegos que favorecen el aprendizaje de las nociones matemáticas}

Los niños, mientras crecen, cambian su manera de jugar de acuerdo con los estadios de desarrollo en el que se encuentren. Por ejemplo, para Piaget los niños en la medida que superan cada estadio o periodos avanzan en su desarrollo al siguiente nivel. Los periodos propuestos por Piaget son: sensoriomotor (entre los 0-2 años), preoperacional (2 y 6 años) y de operaciones concretas (6 a 12 años). Es así que cada etapa constituye un tipo de juego como: funcional o de ejercicio, el simbólico y el juego de reglas.

En el juego funcional, o de ejercicio, predomina el uso del propio cuerpo con el fin de desplazarse de un lugar a otro y así alcanzar los objetos que desea manipular y explorar; en esta etapa que comprende el estadio sensoriomotor, se favorece la comprensión del mundo y la interacción con los objetos. El juego simbólico busca estimular la imaginación y la creatividad en los niños al darle vida a un objeto inanimado o hacer representaciones de algunas situaciones que experimentan en su vida cotidiana. Por último, el juego de reglas está presente en todas las etapas ya mencionadas y permite que el niño se relacione correctamente con los objetos y las personas que intervienen en el juego.

Dentro de los tipos de juego mencionados se encuentra el juego de construcción, en el cual el niño está en la capacidad de manipular los objetos y construir de acuerdo con su interés. Un ejemplo es apilonar bloques hasta formar una torre, de un papel realizar un avión o barco, entre otras cosas que los niños desde su creatividad pueden hacer cuando se obtienen los materiales adecuados para ello.

Cabe resaltar que los niños contemporáneos adquieren habilidades de una manera mucho más rápida por lo que a cierta edad pueden estar en un estadio más avanzado que el planteado por esta teoría, por tal razón, el maestro debe estar dispuesto a los cambios que se presenten en los niños que tiene a cargo de su enseñanza. En esta misma línea, es importante que el maestro de educación inicial reconozca los tipos de juego y los múltiples beneficios que este tiene. Pero esto no se da por inercia; es necesario que las actividades que se desarrollan en el aula de clase estén intencionadas a los objetivos de aprendizaje, al contexto y a las características de los estudiantes. De esta manera, estos aspectos al ser tenidos en cuenta por los maestros pueden brindar el ambiente oportuno que permita un óptimo aprendizaje para los niños por medio de esta estrategia.

Los juegos didácticos y su aporte el desarrollo de los aprendizajes

Desde esta perspectiva, Tirapegui (2008) señala que los juegos didácticos que presenten contenido matemático fomentan una mejor comprensión e interiorización de manera constructiva y divertida para que los niños disfruten de esas clases de manera armoniosa y a su vez productiva. Este disfrute al estar aprendiendo hace que se rompan los esquemas tradicionales, como el que se ha tenido acerca del aprendizaje de las matemáticas, pues es el juego el que marca ese eje diferenciador de lo que comúnmente han sido las clases de matemáticas.

Adicionalmente, es importante tener en cuenta que en la etapa inicial los niños están en constante socialización e interacción en el entorno que le es propio. En otras palabras, las nociones lógico-matemáticas están presentes en la vida del niño en todo momento y en todo lugar. Por esta razón, el docente debe sacar el mejor provecho del entorno que le es familiar al estudiante. Así como seleccionar adecuadamente las actividades y juegos que sean de verdadero interés y estén dirigidos a las 
necesidades de los estudiantes para potenciar las habilidades que los estudiantes han ido adquiriendo o reforzar aquello que el niño no ha logrado aprender completamente, de manera que se logra estimular varios conceptos a la vez (Salvador, 2010).

Lo dicho hasta el momento señala la importancia que tiene el reconocimiento del contexto, el espacio y del juego para la enseñanza de las matemáticas en la etapa preescolar, específicamente en los niños que comprenden las edades de 2 a 4 años. Las acciones lúdicas, de acuerdo con Chacón (2008), deben ser intencionadas y al mismo tiempo atendiendo las necesidades de los niños al momento de jugar.

Además, puede decirse que comúnmente en los jardines infantiles y en los demás grados escolares superiores, la enseñanza de las matemáticas ha estado enmarcada por aspectos tradicionales que poco aportan al goce y al disfrute de estas. Y aunque puede generar aprendizaje este se da de forma mecánica, memorística y pueden contener el goce de aprender y divertirse al mismo tiempo a través del juego. En este sentido, Rodríguez y Muñoz afirman que el aprendizaje de las matemáticas "puede ser una experiencia motivadora si lo basamos en actividades constructivas y lúdicas. El uso de los juegos en la educación matemática es una estrategia que permite adquirir competencias de una manera divertida y atractiva para los alumnos" (2014, p. 1).

Desde esta perspectiva, puede deducirse que las matemáticas están presentes en todos los ámbitos de la vida cotidiana y es por medio de la actividad recreadora que los niños pueden descubrir ese mundo de forma placentera y efectiva. Además, al momento de jugar Rodríguez y Muñoz (2014) afirman que se adquiere un proceso de análisis y se realizan interpretaciones que permite adquirir un aprendizaje más adecuado de las nociones matemáticas.

\section{Conclusiones}

Cabe mencionar que algunos agentes educativos conciben el juego como una forma de entretener a los niños dentro de las aulas de clase. Pues, aunque reconocen que el niño por naturaleza disfruta del juego, es puesto en marcha sin intencionalidad de aprendizaje concreto y, en ocasiones, se observan poco los procesos de socialización y exploración que los niños adquieren al momento de jugar.

Con esta investigación se espera generar reflexión en los docentes y padres para que vean el juego como un mediador del aprendizaje de las nociones lógico matemáticas y aprendan a utilizarlo, más que como una estrategia de diversión para los niños y niñas, como facilitador de habilidades y conocimientos para la vida. Además, que se resignifique la labor que está realizando el docente en las aulas de clase de modo que adquiera nuevos métodos y conocimientos para que el aprendizaje de los niños parta de sus necesidades, intereses y se dé de manera vivencial y experimental. De tal forma que se convierta en algo significativo para la vida.

Es importante retomar que los juegos deben realizarse de acuerdo con el contexto y al espacio donde los estudiantes están inmersos, pues no es lo mismo jugar en zonas urbanas que en las rurales. El docente deberá ser muy creativo al momento de preparar sus planeaciones y actividades lúdicas cuando imparte el aprestamiento lógico matemático en los niños de edad preescolar, debido a que debe ser observador de los juegos que realizan los niños cuando lo hacen de manera libre pero intencionada, con el fin de captar los intereses y preparar una clase acorde a dichos intereses.

Los niños están en la capacidad de aprender lo que el maestro les brinde a través del juego y que desde esta estrategia pedagógica se desarrollan habilidades intelectuales como son los procesos lógico-matemáticos, iniciando con los conceptos propios de la edad en la que se encuentren, por ejemplo, diferenciar colores, nociones espaciales, tamaños, formas, números, entre otros.

Por último, el juego les permite a los niños ser creativos, aprenden a partir de sus propios intereses, les da la oportunidad de aprender y descubrir el mundo a su propio ritmo; asimismo, les da la oportunidad de ir adquiriendo habilidades para la vida. El juego es un reflejo del contexto y la cultura que rodea el niño, por lo tanto, le permite conocerse asimismo y a los otros. 


\section{Referencias}

Blanco, E. V. (s. f.). La lógica matemática en educación infantil. https://uvadoc.uva.es/bitstream/10324/4002/1/TFG-G\%20374.pdf

Bustamante, J. (2004). El desarrollo de la noción de espacio en el niño de educación inicial. https://dialnet.unirioja.es/descarga/articulo/2970459.pdf

Cárdenas, R. y Gómez, D. (2014). El juego en la educación inicial. http://www.colombiaaprende.edu.co/primerainfancia

Camels, D. (2010). El juego corporal: el cuerpo en los juegos de crianza. http://jurecmardelplata. org.ar/wp-content/uploads/2016/03/Eljuegocorporal.pdf

Callejo, M. L. y Vila, A. (2003). Origen y formación de creencias sobre la resolución de problemas. Estudio de un grupo de alumnos que comienzan la educación secundaria. http://emis.ams.org/journals/BAMV/conten/ vol10/mcallejo+vila.pdf

Chacón, P. (2007). El juego didáctico como estrategia de enseñanza y aprendizaje ¿Cómo crearlo en el aula? http://www.e-historia.cl/cursosudla/13-EDU413/lecturas/06\%20-\%20El\%20 Juego $\% 20$ Didactico $\% 20$ Como\%20Estrategia $\% 20$ de $\% 20$ Ense $\%$ C3\%B1anza $\% 20 y \% 20$ Aprendizaje.pdf

Chamoso, J. M., Durán, P. J., García, S. J. F. y Rodríguez, S. M. M. (2004). Análisis y experimentación de juegos como instrumentos para enseñar matemáticas. Suma. https://www.researchgate. net/publication/39222105_Analisis_y_experimentacion_de_juegos_como_instrumentos_ para_ensenar_matematicas

Galeano, E. (2004). El estudio de casos: un interés por la singularidad. En E. Galeano, Estrategias de investigación social cualitativa. El giro en la mirada. Ediciones Carreta.

García, A. y Llull, J. (2009). El juego infantil y su metodología. http://revistapulso.cardenalcisneros. es/documentos/articulos/110.pdf

Grondin, J. (1999). Introducción a la hermenéutica filosófica. Herder.
Ministerio de Educación Nacional (2014). El juego en la educación inicial. http://www.deceroasiempre.gov.co/Prensa/CDocumentacionDocs/ Documento-N22-juego-educacion-inicial.pdf

Muñoz, M. F. y Almonacid, A. (2015). Cognición, juego y aprendizaje: una propuesta para el aula de la primera infancia. Infancia, Educación y Aprendizaje, 1(1), pp. 162-177. https://doi. org/10.22370/ieya.2015.1.1.576

Posada, A, Gómez, J. y Ramírez, H. (2005). El niño sano. Editorial Médica Panamericana.

Quintero, A. S. R., Ramírez, R. L. E. y Jaramillo, V. B. (2016). Actitud lúdica y lenguajes expresivos en la educación de la primera infancia. Revista Virtual Universidad Católica del Norte, 48, 155-170. https://www.redalyc.org/articulo. oa? id=1942/19424590201

Rodríguez, L. y Muñoz, R. (2014). El uso de los juegos como recurso didáctico para la enseñanza y el aprendizaje de las matemáticas: estudio de una experiencia innovadora. http://www.fisem.org/www/union/revistas/2014/39/archivo6.pdf

Salvador, A. (2010). El juego como recurso didáctico en el aula de matemáticas. https://s3.amazonaws.com/academia.edu. documents/37247350/12.Juego.pdf?AWSAccessKeyld=AKIAIWOWYYGZ2Y53UL3A\&EXpires $=1542167668 \&$ Signature=tzPzAp2 I\%2BELB9sJB0fentqptFol\%3D\&response-content-disposition=inline $\% 3 \mathrm{~B} \% 20$ filename $\%$ 3DPorcentajes_Histograma_Poligono_de_frecu.pdf

Schejtman, C., Duhalde, C., Silver, R., Vernengo, M. P., Wainer, M. y Huerin, V. (2009). Los inicios del juego en la primera infancia y su relación con la regulación afectiva diádica y la autorregulación de los infantes. Anuario de Investigaciones, 16, 241-252. https://www.redalyc.org/articulo. oa? id=3691/369139945063

Tirapegui, C. (2008). Eljuegoen la clase de matemáticas. http://www.saber.ula.ve/bitstream/ handle/123456789/20298/articulo12. htm? sequence $=1$ \&isAllowed $=y$ 
Vallejo, S. A. (2009). Juego, material didáctico y ju- Winnicot, D. (1982). Realidad y juego. Gedisa. guetes en la primera infancia. https://educrea. cl/wp-content/uploads/2017/02/DOC2-juego-primera-infancia.pdf

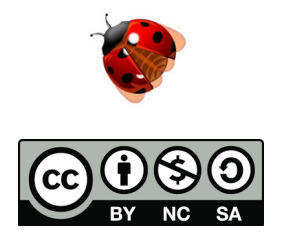

Esta obra está bajo licencia Creative Commons Atribución-NoComercial-Compartirlgual 4.0 Internacional https://creativecommons.org/licenses/by-nc-sa/4.0/deed.es

ISSN 1657-9089 • Vol. 19, no. 2 (julio-diciembre 2020). pp. 133-142 